\title{
Agressão ENTRE PARES NO ESPAÇO VIRTUAL: DEFINIÇÓES, IMPACTOS E DESAFIOS DO CYBERBULLYING
}

\author{
Guilherme Welter Wendt* \\ Carolina Saraiva de Macedo Lisboa**
}

\section{Resumo}

O presente artigo visa apresentar uma revisão da literatura sobre publicaçóes teóricas e empíricas relacionadas ao processo de cyberbullying. Embora existam poucos estudos sobre o tema e haja carência de evidências empíricas na América Latina, o cyberbullying tem sido concebido enquanto uma categoria específica, única e sem precedentes de violência, podendo ser mais abrangente que o fenômeno bullying por ocorrer a qualquer momento e sem um espaço circunscrito e delimitado fisicamente. Os estudos mostram que vítimas de cyberbullying podem estar mais propensas a tentarem suicídio, bem como mais vulneráveis ao desenvolvimento de problemas sociais e emocionais, como a evasão escolar e desempenho acadêmico prejudicado. Além disso, as pesquisas mostram que os envolvidos neste fenômeno apresentam risco aumentado para abuso de substâncias psicoativas e desenvolvimento de sintomas de ansiedade e depressão quando comparados àqueles que não vivenciaram essa forma de agressão entre pares. Assim, aponta-se a importância de um debate atual sobre os aspectos relacionados à prevenção e intervenção em relação ao cyberbullying, envolvendo a família, a escola e também os responsáveis pelo desenvolvimento e execução de políticas públicas.

Palavras-chave: cyberbullying; bullying; agressão; adolescência; geração digital.

* Programa de Pós-Graduação em Psicologia, PUC-RS. Porto Alegre, Rio Grande do Sul, Brasil. E-mail: guilhermewwendt@gmail.com.

** Programa de Pós-Graduação em Psicologia, PUC-RS. Porto Alegre, Rio Grande do Sul, Brasil.Email: carolina.lisboa@pucrs. 


\title{
74 - Cyberbullying
}

\begin{abstract}
PeERS AGgRESSION IN THE VIRTUAL SPACE: DEFINITIONS, IMPACTS, AND CHALLENGES OF CYBERBULLYING

The present paper aims to present a literature review on empirical and theoretical studies about cyberbullying process. Although there are few studies about this theme and there is almost no empirical evidence found in Latin America, cyberbullying has been defined as a specific phenomenon, unique and without violence antecedents. Cyberbullying is directly related to bullying but it can happen any time and does not necessarily take place in a natural environment. Researches have shown that cyberbullying victims are more vulnerable to commit suicide than other youth, as well as to develop social and emotional problems that can lead to school dropout and poor academic achievement. Besides all, studies also evidenced that youth involved in this phenomenon are at risk for substance use and abuse as well as to present depression and anxiety symptoms when compared to those youth that do not experienced this kind of violence. The update discussion about cyberbullying is important especially focusing on prevention and intervention programs involving family (parents), school and public police makers.

Keywords: cyberbullying; bullying; aggression; adolescence; digital generation.
\end{abstract}

\section{Resumen}

Agresión ENTRE PAREs EN EL ESPACIO VIRTUAL: DEFINICIONES, IMPACTOS Y DESAFÍOS DE CIBERBULLYING

En este articulo se presenta una revisión de la literatura sobre las publicaciones teóricas y empiricas relacionadas con el proceso de ciberbullying. Aunque hay pocos estudios sobre el tema y hay una falta de evidencia empirica en América Latina, el ciberbullying ha sido diseñado como una categoría especifica, la violencia única y sin precedentes puede ser más amplio que la ocurre en el fenómeno bullying por ocurrir en cualquier momento y sin delimitado espacio circunscrito físicamente. Los estudios muestran que las víctimas de ciberbullying pueden ser más propensos a intentar el suicidio, así como más vulnerables a la aparición de problemas sociales y emocionales tales como el absentismo escolar y el rendimiento académico prejuicios. Además, la investigación muestra que las personas involucradas en este fenómeno tienen un mayor riesgo de abuso de sustancias y de aparición de los sintomas de ansiedad y depresión en comparación con aquellos que no experimentan esta forma de agresión entre compañeros. De este modo, este artículo señala la importancia de un debate en curso sobre los aspectos relacionados con la prevención e intervención en relación con el ciberbullying, con la participación de la familia, la escuela y también los responsables de la elaboración y ejecución de politicas públicas.

Palabras clave: ciberbullying; bullying; agresión; adolescencia; generación digital.

Psic. Clin., Rio de Janeiro, vol. 25, N.I, P. $73-87,2013$ 


\section{Introdução}

Especialmente na última década, observa-se que o conceito de violência e agressividade vem sendo ampliado e analisado de forma contextualizada. Esforços de pesquisadores em compreender as diferentes manifestaçōes e funções do comportamento agressivo, bem como em desenvolver modelos explicativos específicos para diferentes culturas, aparecem sob a forma de pesquisas empíricas e revisóes teóricas (Berger \& Lisboa, 2009; Smith, Smith, Osborn \& Samara, 2008; Walker, 2010). Neste sentido, as formas de agressão relacional e indiretas de comportamento agressivo, ambas prejudiciais à saúde física e psicológica dos indivíduos, também ganham atenção (Berger \& Lisboa, 2009; Marsh, Parada, Yeung \& Healey, 2001).

Como um subtipo de comportamento agressivo com diferentes formas de manifestação, o processo de bullying intriga e desafia profissionais de distintas áreas, assim como autoridades envolvidas no desenvolvimento de políticas públicas. Descrito na literatura especializada já no início do século passado, pelo primeiro presidente da American Psychological Association, Stanley G. Hall (1904), o bullying, atualmente, tem mobilizando educadores, pais e alunos no mundo inteiro. Quase um século depois, o fenômeno passaria a ser estudado de modo sistematizado, sendo que o psicólogo norueguês Dan Olweus foi um dos primeiros a delimitar e explicitar o conceito para o processo de bullying. Segundo Olweus (1993), bullying é uma ação de violência sistemática, desigual e recorrente no âmbito escolar na qual se identifica um agressor que tem a intenção de causar dano a alguém (vítima), que se encontra, normalmente, com pouco ou nenhum recurso de revidar.

O processo de bullying é influenciado pela interação dinâmica com o ambiente no qual nascem e se desenvolvem os jovens, conforme destacam Lisboa e Koller (2004). No contexto brasileiro, uma recente investigação indicou que aproximadamente $30 \%$ dos estudantes do ensino fundamental foi vítima de bullying (Malta et al., 2010). Comumente observa-se a agressão física e os insultos verbais, bem como as agressóes indiretas (como o isolamento) em momentos como o recreio, sendo que estes comportamentos são, geralmente, motivados e mantidos por sentimentos como inveja, preconceito e intolerância. Do mesmo modo, este processo é, muitas vezes, sutil aos olhos de professores e funcionários da escola e, ainda, pode ter o seu início a partir de uma brincadeira inofensiva, uma "chacota" que, a partir do reforçamento e repetiçáo, traz implicaçóes nocivas diversas tanto para os autores como para as vítimas e também ao subgrupo dos expectadores (Hoff \& Mitchel, 2009; Lisboa, Braga \& Ebert, 2009).

Psic. Clin., Rio de Janeiro, vol. 25, N.I, P. $73-87,2013$ 
Paralelo a esta conscientização e maior atenção aos casos de violência nas escolas e também à constatação de maiores frequências destes comportamentos, observa-se também um rápido aumento da agressão através de meios eletrônicos de comunicação e interação. A incorporação da tecnologia e sua utilização em casa e na escola deflagra preocupaçôes a respeito de tópicos como privacidade, segurança de informaçôes e outros riscos, como exposição a conteúdo indesejado, pornografia, publicidade ofensiva, manifestaçôes de ódio, racistas, homofóbicas, etc. Comportamentos agressivos no contexto virtual podem ser descritos como cyberbullying ou bullying virtual (Bullen \& Harré, 2000; Valkenburg \& Peter, 2011). Este fenômeno, conforme Shariff (2011), também pode ser chamado de bullying eletrônico ou assédio online.

Dessa maneira, o presente estudo visa apresentar uma revisão da literatura sobre publicaçôes relacionadas ao cyberbullying, suas características, impactos e desafios. Os estudos foram recuperados a partir de pesquisas feitas nas principais bases de dados nacionais e internacionais (PubMed, Academic Search Premier, Science Direct, Pepsic, Scielo e Biblioteca Virtual em Saúde), no período compreendido entre o ano de 2000 e o ano de 2012. Como principal estratégia de pesquisa, foram utilizados os descritores "cyberbullying", "cyber bullying" e "bullying virtual". Além disso, foi realizada uma análise qualitativa nas referências bibliográficas dos estudos incluídos nesse artigo, com vistas à obtençáo de fontes complementares que, por algum motivo, não foram recuperadas durante as pesquisas nas bases de dados. Obras impressas de referência para o estudo da agressão entre pares durante a infância e adolescência também foram incluídas no presente estudo. Deste modo, organizar-se-á essa discussão apresentando-se inicialmente a natureza e o contexto no qual o cyberbullying emerge, focando nos impactos às vítimas e aos agressores; em seguida, serão abordados os fatores relacionados à ocorrência desse tipo de comportamento na contemporaneidade. Finalmente, serão debatidos aspectos que possam resultar em açôes preventivas e interventivas na família e na escola.

\section{Contextos de agressão: compreendendo o universo dos nativos digitais}

O termo "nativo digital" não soa de todo modo estranho aos profissionais da psicologia e ciências afins. Afinal, ele se refere à primeira geração conectada à rede mundial de computadores, que teve seu início por volta dos anos oitenta do século passado. Esse contingente expressivo de crianças e adolescentes está crescendo em um contexto mediado cada vez mais pelo uso de tecnologias de

Psic. Clin., Rio de Janeiro, vol. 25, N.I, P. $73-87,2013$ 
informação e comunicação, reverberando em manifestaçôes psicossociais diversificadas (Palfrey \& Gasser, 2011). Conforme aponta Gross (2004), do Instituto Infantil de Mídia Digital da Universidade da Califória, os adolescentes de hoje são flexíveis e expõem sua identidade em múltiplos contextos digitais, como os fóruns, as salas de bate-papo, blogs, entre outros. $\mathrm{Na}$ medida em que as crianças e adolescentes intensificam a interação com as novas tecnologias de interação e aumentam sua participação em sites de redes sociais, criando perfis públicos e compartilhando informaçóes pessoais, novos dispositivos tecnológicos são criados em resposta a essa demanda.

Exemplo de tal fato pode ser percebido através da notória proliferação de redes de relacionamento de modo tâo marcante e rápido que aparelhos celulares já estão aptos à conexão imediata e em praticamente qualquer lugar, à disposição dos usuários durante 24 horas por dia, sete dias por semana. Assim, na atualidade, um dos modos para a expressão da identidade e interação com pares é perpassado pela "paisagem digital" (Palfrey \& Gasser, 2011, p. 76). Essa nova configuraçáo do mundo relacional e do espaço de interaçáo social se faz presente também na realidade de grande parte dos jovens brasileiros, gerando novas representaçóes mentais acerca de si mesmos e do mundo que os cerca (Estefanon \& Eisenstein, 2008).

Do mesmo modo, seguindo as transformações decorrentes da onipresença das novas tecnologias de interaçáo, formas únicas para a expressão do comportamento agressivo entre pares emergem, Steffgen, König, Pfetsch e Melzer (no prelo) sumarizam que "os agressores virtuais têm uma percepçáo singular de controle e apresentam crenças de imunidade em relação a sanções de seus atos". Esta afirmação polêmica e de certa forma taxativa remete à importância de estudos descritivos, revisões de literatura sobre o tema e ressignificação de crenças e "pré-conceitos". Com efeito, estudos de natureza teórica conduzidos no Brasil também indicam que o ambiente virtual pode ser propício para a manifestação de comportamentos agressivos (Lisboa \& Ebert, 2012; Stelko-Pereira \& Williams, 2010; Wendt, Campos \& Lisboa, 2010), bem como indicam a importância da realização de estudos empíricos no país com o foco nessa problemática (Lisboa, Braga \& Ebert, 2009).

\section{Cyberbullying: impactos e desafios endereçados aos pais e professores da geraçáo digital}

Neste contexto do século XXI e do mundo globalizado, a violência moral, física e relacional passou a não se limitar somente ao contexto da escola ou outros

Psic. Clin., Rio de Janeiro, vol. 25, N.I, P. $73-87,2013$ 
espaços fisicamente circunscritos (Juvonen \& Gross, 2008; Kiriakidis \& Kavoura, 2010). Ou seja, parece que os jovens, atualmente, vêm aos poucos adotando uma nova forma de praticar o bullying, o que muda qualitativamente as regras tradicionais da agressão entre pares (Brown, Jackson \& Cassidy, 2006). O cyberbullying, por definição, compreende o uso de ferramentas tecnológicas para assediar, ameaçar, constranger ou humilhar outra pessoa, simular ou tentar violar senhas das vítimas (Juvonen \& Gross, 2008).

As formas mais comuns de cyberbullying ocorrem, geralmente, através do envio de e-mails, mensagens de texto, divulgação de fotos e vídeos ofensivos, manipulação de imagens, insultos em salas de bate-papo ou em redes sociais, que podem ser anônimos e atingir um público infinito de expectadores em pouco tempo (Brown, Jackson \& Cassidy 2006; Shariff, 2011). Quanto as suas características, sabe-se que as crianças escolhidas como vítimas são, na sua maioria, identificadas como mais frágeis, com pouco ou nenhum recurso de enfrentamento e, não raro, os atos de agressão online contra estas envolvem o uso de linguagem depreciativa, com conotaçóes sexuais, de ódio e ameaça (Varjas, Henrich \& Meyers, 2009). Ademais, esses ataques adquirem um caráter de permanência, diferentemente do bullying, uma vez que usuários no mundo inteiro podem, a qualquer tempo, assistir, compartilhar e salvar em seus computadores manifestos de cyberbullying (Smith, 2012).

Uma vítima de cyberbullying pode nunca saber o minuto quando será atacada novamente, pois a internet permite ao agressor recorrer à vítima de inúmeras formas e a qualquer momento, alterando as delimitaçóes contextuais do bullying na escola. Ao contrário da vítima de bullying, que sabe que será atacada quando chegar à escola ou teme pela hora do recreio, uma vítima de cyberbullying pode receber mensagens de texto com ameaças inclusive enquanto estiver dormindo. Os xingamentos e insultos, comuns no bullying, são também uma das formas mais utilizadas pela agressão virtual, sendo que programas de mensagens instantâneas vêm sendo usados de modo recorrente (Juvonen \& Gross, 2008), assim como sites de redes sociais (Kowalski \& Limber, 2007).

Além disso, é pertinente incluir no conjunto de dimensóes que perpassam o cyberbullying que, no ciberespaço, a capacidade de colocar-se no lugar do outro pode ficar prejudicada (Smith, 2012). Kowalski e Limber (2007) salientam que, protegidos por inúmeros recursos tecnológicos, os agressores podem potencializar as suas açôes contra outra pessoa, com prejuízos na avaliação e no sentimento da consequência de seus atos. Ou seja, diferente do contexto no qual se processam as interaçôes face a face, no qual é possível a identificação e modulação dos comportamentos de modo contingencial, os jovens envolvidos nas formas de agressão online têm prejuízo no desenvolvimento e manutençấo de algumas habilidades

Psic. Clin., Rio de Janeiro, vol. 25, N.I, P. $73-87,2013$ 
sociais (Kowalski \& Limber, 2007; Steffgen et al., no prelo). Em outras palavras, um dos riscos que o cyberbullying pode oferecer aos jovens é o déficit no desenvolvimento de sua capacidade empática.

Em um inquérito online envolvendo crianças e adolescentes de 12 a 17 anos, cerca de três quartos dos participantes foram identificados com a presença de pelo menos um incidente de cyberbullying no último ano, sendo que, desse grupo, quase $85 \%$ também eram vítimas de bullying (Juvonen \& Gross, 2008). Este dado confirma e sustenta as afirmaçóes do estudo de Steffgen $e t$ al. (no prelo) de que o cyberbullying pode ser mais prevalente do que o bullying tradicional nos dias atuais.

Li (2007) que, em um estudo com alunos da sétima série do ensino fundamental na China, encontrou prevalência de $25 \%$ de cyberbullying, sendo que a maioria (60\%) das vítimas era do sexo feminino e $52 \%$ dos cyberbullies (perpetradores) eram do sexo masculino, o que corrobora achados de outros estudos sobre agressividade em geral e bullying (Huang \& Chou, 2010; Li, 2007; 2008). Patchin e Hinduja (2010) conduziram um estudo com o objetivo de verificar quais os impactos do cyberbullying em jovens. Os pesquisadores estudaram 1.963 estudantes do ensino médio, provenientes de 30 escolas americanas. Através de um instrumento de autorrelato, estes profissionais verificaram que tanto os cyberbullies como as vítimas apresentaram níveis baixos de autoestima em maior frequência quando comparados aos alunos sem história de vitimização online. Ademais, 23,1\% da amostra declararam ter sido perpetrador e $18,8 \%$ ter sido vítima de cyberbullying. No tocante às questóes de gênero, diferenças bem demarcadas foram identificadas na ocorrência dos subtipos de vitimização virtual entre meninos e meninas.

Além disso, há indícios apontando que adolescentes vítimas de cyberbullying podem estar mais propensos a tentar suicídio do que aqueles que não experimentam essas formas de agressão entre pares (Cassidy, Jackson \& Brown, 2009). Na pesquisa de Patchin \& Hinduja (2010), foi constatado que aqueles participantes envolvidos com o bullying ou cyberbullying, tanto como perpetradores como na condição de vítimas, apresentaram maiores frequências de ideação suicida e também se mostraram mais propensos a tentar suicídio em comparação aos estudantes que não haviam sido expostos a essas formas de agressão.

No tocante às diferenças entre os sexos no envolvimento com cyberbullying, a pesquisa recente de Schoffstall e Cohen (2011) indicou que meninos e meninas não diferem significativamente na frequência de condutas agressivas através da internet. O estudo, que contou com a participação de 192 estudantes, acrescentou ainda outros importantes conhecimentos sobre os impactos da vitimização "tradicional" e eletrônica. Por exemplo, os participantes envolvidos com o cyber- 
bullying apresentaram correlação superior com sentimentos de solidão $(\mathrm{r}=0,30$, $\mathrm{p}<0,01)$ quando comparados aos estudantes envolvidos com o bullying $(\mathrm{r}=0,23$, $\mathrm{p}<0,01)$. Além disso, na medida em que aumentam os episódios de cyberbullying, observa-se também o acréscimo de uso de meios eletrônicos de interação $(\mathrm{r}=0,50$, $\mathrm{p}<0,01)$. Já a investigação de $\mathrm{Li}(2006)$ encontrou resultados discordantes entre meninos e meninas em relação ao cyberbullying. $\mathrm{O}$ estudo teve como amostra 264 estudantes canadenses selecionados aleatoriamente. Um em cada quatro participantes reportou ter sido vítima de cyberbullying, sendo que 22\% dos agressores virtuais eram do sexo masculino, contra $12 \%$ do sexo feminino. Em relação à vitimização online, todavia, não foram constatadas diferenças significativas entre meninos (25\%) e meninas (25,6\%).

Em um estudo realizado em Luxemburgo, pesquisadores verificaram que os agressores do bullying escolar tendem a ser também os cyberbullies no mundo virtual e que, ao mesmo tempo, as vítimas de bullying também tendem a ser vítimas de cyberbullying (Steffgen et al., no prelo). Deste modo, é possível pensar em um processo constante e cíclico de agressão, altamente deletério à saúde dos jovens em desenvolvimento nos mais diversos contextos. Ao mesmo tempo, também é possível pensar que as vítimas do bullying possam praticar o cyberbullying motivadas pela retaliação dos seus agressores na vida "real".

Ainda em relação ao foco de estudos sobre os impactos e características do fenômeno, observa-se que sintomas depressivos podem mediar o processo de $c y$ berbullying (Luk, Wang \& Simons-Morton, 2010; Smith, Mahdavi, Carvalho, Fisher, Russell \& Tippett, 2008), bem como a associação deste processo com o uso de substâncias psicoativas (Goebert, Else, Matsu, Chung-Do \& Chang, 2010). A literatura evidencia também uma relação entre a exposição ao cyberbullying e o tempo gasto na internet, identificando que quanto mais tempo o jovem interage virtualmente, maiores suas chances de sofrer cyberbullying (Hetzel-Riggin \& Pritchard, 2011; Twyman, Saylor, Taylor \& Comeaux, 2010). Além disso, adolescentes vítimas de cyberbullying reportaram, na investigação de Mesch (2009), maior uso de telefones celulares para a comunicação com seus pares.

O computador, sendo de uso particularmente individual, torna o controle parental prejudicado e, se pensarmos que os maiores experts em tecnologia são justamente os filhos, e não seus pais, a questáo assume significativa complexidade (Mesch, 2009). Assim, investigaçôes também vêm sendo realizadas com o objetivo de analisar a influência do controle dos pais sobre o uso da tecnologia por parte dos filhos (Mesch, 2009; Valcke, Bonte, De Wever \& Rots, 2010).

As crianças e adolescentes que recebem supervisão dos pais enquanto navegam, ou que declaram a existência de regras claras quanto ao uso da tecnologia,

Psic. Clin., Rio de Janeiro, vol. 25, N.I, P. 73-87, 2013 
vêm apresentando comportamentos de risco em menor intensidade (Fleming, Greentree, Cocotti-Muller, Elias \& Morrison, 2006; Heim, Brandtzæg, Endestad, Kaare \& Torgersen, 2007). Heim et al. (2007), em um estudo exploratório com adolescentes na Noruega, observaram que o uso de mídia costuma ser mais criativo e saudável quando os pais estâo presentes fisicamente. Logo, essas práticas de supervisão e presença física devem ser incorporadas ao repertório comportamental dos pais e professores responsáveis pela segurança da geração digital (Diamanduros, Downs \& Jenkins, 2008; Dooley, 2010; Hinduja \& Patchin, 2007, 2010).

Considerando-se esses aspectos, pesquisadores salientam que pais, professores, gestores públicos e os profissionais da área da saúde devem ser informados sobre os comportamentos de risco no ambiente virtual e sobre os impactos no desenvolvimento psicossocial de modo a melhor avaliar os riscos aos quais está suscetível a população mais jovem (Shariff, 2011; Walker, 2010). Conforme descrito anteriormente, a agressão entre pares é um problema social e educacional que aparece na escola e em outros contextos, como no próprio lar. Assim, o crescimento dos casos de cyberbullying evidencia para as escolas e as famílias a necessidade de desenvolvimento de novas competências, bem como apontam para a urgência de políticas públicas de regulação e intervenção diante do fenômeno (Palfrey \& Gasser, 2011; Walker, 2010).

\section{Consideraçóes finais}

Esta revisão teve o intuito de definir e levantar algumas características e consequências do cyberbullying, salientando os riscos ao desenvolvimento humano saudável. Também foi objetivo pensar sobre aspectos que possam representar ações preventivas e focais, como a supervisão parental adequada (Smith, Smith, Osborn \& Samara, 2008). Foram discutidos os resultados dos estudos que descrevem essa nova forma de agressão entre pares e buscam identificar os fatores que possivelmente contribuem para uma maior vitimização online e os prejuízos envolvidos (Diamanduros, Downs \& Jenkins, 2008; Dooley, 2010; Hetzel-Riggin \& Pritchard, 2011; Hinduja \& Patchin, 2008, 2010; Luk, Wang \& Simons-Morton, 2010; Valcke, Schellens, Van Keer \& Gerarts, 2007).

A vitimização online emergiu como um fator de risco adicional para o desenvolvimento de sintomas de depressão e ansiedade em adolescentes envolvidos em atos de bullying (Juvonen \& Gross, 2008), bem como para o envolvimento em relaçóes com "pares fora do padrão" (Shariff, 2011, p. 53), comprometendo, assim, o desenvolvimento psicossocial de um modo amplo 
(Valkenburg \& Peter, 2011). Cerca de 25\% das vítimas de cyberbullying náo buscam atendimento especializado e esse afastamento da clínica pode causar prejuízos sérios, ou mesmo irreversíveis, como nos casos extremos de suicídios, homicídios e massacres em escolas (Price \& Dalgleish, 2010). Desse modo, as repercussóes do fenômeno para as vítimas, famílias e escolas, discutidas nesse artigo, representam desafios cotidianos que são impostos aos terapeutas, educadores e responsáveis pela implementação de políticas públicas, que necessitam de forma urgente compreender as especificidades subjetivas e emocionais dos jovens sob a influência do "universo digital", que permeia grande parte desta geração de crianças e adolescentes.

Embora não possamos negar a importância da tecnologia para fins educativos, de lazer e informação, o anonimato que perpassa muitos dos atos de agressão virtual, associado à rapidez com que ocorrem, serve de sustentaçấo para que se mencione a gravidade do assunto. É preciso encontrar um ponto de equilíbrio entre as responsabilidades pessoais e a liberdade que as novas tecnologias podem oferecer. As escolas devem rever suas políticas de uso de ferramentas tecnológicas, com regras claras sobre a entrada de equipamentos eletrônicos, como smartphones, notebooks e similares. Avisos em telas dos computadores institucionais devem alertar sobre as atitudes que são intoleráveis dentro do espaço institucional, visando educar os alunos sobre assertividade e ética também no uso das tecnologias e nas relaçôes virtuais (Goebert et al., 2010; Walker, 2010).

A omissão e negligência dos pais e professores diante desse novo contexto de interação dos jovens pode afetar o desempenho escolar do aluno e influenciar negativamente no clima escolar de um modo geral (Williams \& Guerra, 2007). $\mathrm{Na}$ maioria dos países, as escolas são aconselhadas a não permitirem uso livre da internet ou mesmo a monitorar a sua utilização, embora a proibição não seja a solução única, uma vez que, complementarmente, os professores precisam intervir nos casos de cyberbullying com propriedade em suas orientaçôes (Shariff, 2011).

Antes de optar por uma postura impositiva e restritiva acerca do uso da tecnologia, é mais importante intervir preventivamente. Desse modo, um ambiente escolar positivo, no qual os professores expressem apoio emocional aos alunos, pode ser muito eficaz para a prevenção do cyberbullying e, inclusive, do comportamento suicida (Hinduja \& Patchin, 2007; 2008; 2010). Convém salientar que a prevenção efetiva somente pode ser possível quando houver, de fato, uma real dimensão da representação mental deste novo universo que se apresenta aos jovens, com suas linguagens, símbolos e motivaçóes peculiares (Smith, 2012).

Além disso, os indicativos de proteção dos jovens incluem, necessariamente, uma atitude proativa e vigilante por parte dos pais e responsáveis. Os

Psic. Clin., Rio de Janeiro, vol. 25, N.I, P. 73-87, 2013 
pais precisam estar mais próximos dos seus filhos, orientando e acompanhando pessoalmente o que o jovem realiza na rede, com quem fala e quanto tempo despende online. "A supervisão dos pais está fortemente associada a um decréscimo na exposição a conteúdo inapropriado do ciberespaço" (Valcke et al. 2007, p. 2843). Ao mesmo tempo, esta presença física dos pais não pode ser permissiva ou invasiva, mas partir de um estilo parental assertivo (Valcke et al., 2010; Valcke et al., 2007).

Embora as características dos tradicionais participantes bullying já tenham sido identificadas e estudadas durante alguns anos, a investigação sobre o cyberbullying ainda é muito recente (Bullock, Wong-Lo \& Gable, 2011; Couvillon \& Ilieva, 2011; Goebert et al., 2010; Lisboa \& Ebert, 2012). Alguns estudos vêm situando o cyberbulling enquanto uma categoria específica, única e sem precedentes de violência (Varjas, Henrich \& Meyers, 2009; Valkenburg \& Peter, 2011), podendo ser mais abrangente que o bullying porque ocorre a qualquer momento e sem um espaço circunscrito. É importante que os profissionais compreendam que o cyberbullying é um processo que ocorre em adição ao bullying como um subtipo de bullying com características específicas (Stelko-Pereira \& Williams, 2010). Em muitos casos, pode não haver o bullying, mas pode estar ocorrendo a vitimização online; portanto, trata-se de processos sobrepostos, embora independentes.

Em síntese, as crianças e adolescentes de hoje demandam novos olhares e novas práticas clínicas, educativas e parentais. Assim, antes de sugerir causalidade ou "eleger" culpados para punição, deve-se pôr em pauta e estimular pesquisas sobre um assunto que vem sendo manchete de inúmeros veículos da mídia em geral. Até o presente momento, as investigaçóes científicas e evidências empíricas são escassas e, na América Latina, praticamente inexistentes (Lisboa, Braga \& Ebert, 2009). Finalmente, sugere-se a condução de estudos em diferentes culturas, que podem não apenas ajudar na identificação de impactos precisos do cyberbullying e dos modos para enfrentá-lo, mas também apontar para características distintas para vítimas e agressores, abarcando a complexidade deste processo (Valkenburg \& Peter, 2011).

\section{Referências}

Berger, C. \& Lisboa, C. (2009). Violencia escolar: estudios y posibilidades de intervención en Latinoamérica. Santiago: Editorial Universitária.

Brown, K., Jackson, M. \& Cassidy, W. (2006). Cyber-bullying: developing policy to direct responses that are equitable and effective in addressing this special form of bullying. Canadian Journal of Educational Administration and Policy, 57(1), 1-35.

Psic. Clin., Rio de Janeiro, vol. 25, N.I, P. $73-87,2013$ 


\section{4 - Cyberbullying}

Bullen, P. \& Harré, N. (2000). The Internet: it's effects on safety and behavior, implications for adolescents. Department of Psychology, University of Auckland. Recuperado em 3 de maio, 2011, de <http://www.netsafe.org.nz/Doc_Library/patbullen.pdf>.

Bullock, L. M., Wong-Lo, M. \& Gable, R. A. (2011). Cyberbullying: what is it and how can we combat it? Preventing School Failure, 55(2), 62-63.

Cassidy, W., Jackson, M. \& Brown, K. N. (2009). Sticks and stones can break my bones, but how can pixels hurt me? Students' experiences with cyber-bullying. School Psychology International, 30(4), 383-402.

Couvillon, M. A. \& Ilieva, V. (2011). Recommended practices: a review of Schoolwide Preventative programs and strategies on Cyberbullying. Preventing School Failure, 55(2), 96-101.

Diamanduros, T., Downs, E. \& Jenkins, S. J. (2008). The role of school psychologists in the assessment, prevention, and intervention of cyberbullying. Psychology in the Schools, 45(8), 693-704.

Dooley, J. J. (2010). Cyber-bullying: Legal implications and strategies for schools. Journal of CyberTherapy and Rehabilitation, 3(4), 428-430.

Estefanon, S. G. B. \& Eisenstein, E. (2008). Geração digital: riscos e beneficios das novas tecnologias para crianças e adolescentes. Rio de Janeiro: Vieira e Lent.

Fleming, M., Greentree, S., Cocotti-Muller, D., Elias, K. \& Morrison, S. (2006). Safety in Cyberspace - adolescents' safety and exposure online. Youth Society, 38(2), 135-154.

Goebert, D., Else, I., Matsu, C., Chung-Do, J. \& Chang, J. Y. (2010). The Impact of Cyberbullying on substance use and mental health in a multiethnic sample. Maternal and Child Health Journal, 8(1), 1-5.

Gross, E. (2004). Adolescent internet use: what we expect, what teens report. Journal of Applied Developmental Psychology, 25(6), 633-649.

Hall, S. G. (1904). Adolescence: it's psychology and its relations to Physiology, Anthropology, Sociology, Sex, Crime, Religion, and Education. New York: Appleton.

Heim, J., Brandtzæg, P. B., Endestad, T., Kaare, B. H. \& Torgersen, L. (2007). Children's usage of media technologies and psychosocial factors. New Media \& Society, 9(3), 425-54.

Hetzel-Riggin, M. D. \& Pritchard, J. R. (2011). Predicting problematic internet use in men and women: the contributions of psychological distress, coping style, and body esteem. Cyberpsychology, Behavior, and Social Networking, 14(9), 519-525.

Hinduja, S. \& Patchin, J. W. (2007). Offline consequences of online victimization: School violence and delinquency. Journal of School Violence, 6(3), 89-112.

Hinduja, S. \& Patchin, J. W. (2008). Cyberbullying: an exploratory analysis of factors related to offending and victimization. Deviant Behavior, 29(2), 129-156.

Hinduja, S. \& Patchin, J. W. (2010). Bullying, cyberbullying, and suicide. Archives of Suicide Research, 14(3), 206-221.

Psic. Clin., Rio de Janeiro, vol. 25, N.I, P. $73-87,2013$ 
Hoff, D. L. \& Mitchell, S. N. (2009). Cyberbullying: causes, effects, and remedies. Journal of Educational Administration, 47(5), 652-665.

Huang, Y. \& Chou, C. (2010). An analysis of multiple factors of cyberbullying among junior high school students in Taiwan. Computers in Human Behavior, 26(6), 1581-1590.

Juvonen, J. \& Gross, E. F. (2008). Extending the school grounds? Bullying Experiences in Cyberspace. Journal of School Health, 78(9), 496-505.

Kiriakidis, S. P. \& Kavoura, A. (2010). Cyberbullying: a review of the literature on harassment through the internet and other electronic means. Family and Community Health, 33(2), 82-93.

Kowalski, R. M. \& Limber, S. P. (2007). Electronic bullying among middle school students. Journal of Adolescent Health, 41(6), S22-S30.

Li, Q. (2006). Cyberbullying in schools: A research of gender differences. School Psychology International, 27(2), 157-170.

Li, Q. (2007). New bottle but old wine: a research of cyberbullying in schools. Computers in Human Behavior, 23(4), 1777-1791.

$\mathrm{Li}, \mathrm{Q}$. (2008). A cross-cultural comparison of adolescents' experience related to cyberbullying. Educational Research, 50(3), 223-234.

Lisboa, C. \& Koller, S. H. (2004). Interações na escola e processos de aprendizagem: fatores de risco e proteção. In: J. A. Bzuneck \& E. Boruchovitch (org.). Aprendizagem: processos psicológicos e contexto social na escola (pp. 201-224). Petrópolis: Vozes.

Lisboa, C. S. M., Braga, L. \& Ebert, G. (2009). O fenômeno bullying ou vitimização entre pares na atualidade: definiçóes, formas de manifestação e possibilidades de intervençáo. Contextos Clínicos, 2(1), 59-71.

Lisboa, C. S. M. \& Ebert, G. (2012). Violência na escola: reflexões sobre as causas e propostas de açóes preventivas focais. In: L. F. Habigzang \& S. H. Koller (org.), Violência contra crianças e adolescentes: Teoria, pesquisa e prática (pp. 190-202). Porto Alegre: ArtMed.

Luk, J. W., Wang, J. \& Simons-Morton, B. (2010). Bullying victimization and substance use among U.S. adolescents. Prevention Science, 11(4), 355-359.

Malta, D. C., Silva, M., Mello, F. C., Monteiro, R. A., Sardinha, L. M., Crespo, C., Carvalho, M. G., Silva, M. M. \& Porto, D. L. (2010). Bullying nas escolas brasileiras: resultados da Pesquisa Nacional de Saúde do Escolar (PeNSE), 2009. Ciência \& Saúde Coletiva, 15(2), 3065-3076.

Marsh, H., Parada, R., Yeung, A. \& Healey, J. (2001). Aggressive school troublemakers and victims: A longitudinal model examining the pivotal role of self-concept. Journal of Educational Psychology, 93(2), 411-419.

Mesch, G. S. (2009). Parental mediation, online activities, and cyberbullying. CyberPsychology \& Behavior, 12(4), 387-393.

Olweus, D. (1993). Bullying at school: What we know and what we can do. London: Blackwell.

Psic. Clin., Rio de Janeiro, vol. 25, N.I, P. $73-87,2013$ 
Palfrey, J. \& Gasser, U. (2011). Nascidos na era digital: entendendo a primeira geração de nativos digitais. Porto Alegre: ArtMed.

Patchin, J. W. \& Hinduja, S. (2010). Cyberbullying and self-esteem. Journal of School Health, 80(12), 614-621.

Price, M. \& Dalgleish, J. (2010). Cyberbullying experiences, impacts and coping strategies as described by Australian young people. Youth Studies Australia, 29(2), 51-59.

Schoffstall, C. L. \& Cohen, R. (2011). Cyber aggression: the relation between online offenders and offline social competence. Social Development, 20(3), 587-604.

Shariff, S. (2011). Ciberbullying: questōes e soluçóes para a escola, a sala de aula e a família. Porto Alegre: ArtMed.

Smith, P. K., Mahdavi, J., Carvalho, M., Fisher, S., Russell, S. \& Tippett, N. (2008). Cyberbullying: its nature and impact in secondary school pupils. Journal of Child Psychology and Psychiatry, 49(4), 376-385.

Smith, P. K., Smith, C., Osborn, R. \& Samara, M. (2008). A content analysis of school anti-bullying policies: Progress and limitations. Educational Psychology in Practice, 24(1), $1-12$.

Smith, P. K. (2012). Cyberbullying and cyber aggression. In: S. R. Jimerson, A. B. Nickerson, M. J. Mayer \& M. J. Furlong (org.), Handbook of school violence and school safety: international research and practice (pp. 93-103). New York: Routledge.

Steffgen, G., König, A., Pfetsch, J. \& Melzer, A. (no prelo). The role of empathy for adolescents' cyberbullying behaviour. Kwartalnik Pedagogiczny.

Stelko-Pereira, A. C. \& Williams, L. C. A. (2010). Reflexôes sobre o conceito de violência escolar e a busca por uma definição abrangente. Temas em Psicologia, 18(1), 45-55.

Twyman, K., Saylor, C., Taylor, L. A. \& Comeaux, C. (2010). Comparing children and adolescents engaged in cyberbullying to matched peers. Cyberpsychology, Behavior, and Social Networking, 13(2), 195-199.

Valcke, M., Schellens, T., Van Keer, H. \& Gerarts, M. (2007). Primary school children's safe and unsafe use of the Internet at home and at school: an exploratory study. Computers in Human Behavior, 23, 2838-2850.

Valcke, M., Bonte, S., De Wever, B. \& Rots, I. (2010). Internet parenting styles and the impact on Internet use of primary school children. Computers \& Education, 55(2), 454-464.

Valkenburg, P. M. \& Peter, J. (2011). Adolescents' online communication: an integrated model of its attraction, opportunities, and risks. Journal of Adolescent Health, 48, 121-127.

Varjas, K., Henrich, C. C. \& Meyers, J. (2009). Urban middle school students' perceptions of bullying, cyberbullying, and school safety. Journal of School Violence, 8(2), 159-176.

Walker, H. M. (2010). Relational Aggression in Schools: Implications for future research on screening intervention and prevention. School Psychology Review, 39(4), 594-600.

Psic. Clin., Rio de Janeiro, vol. 25, N.I, P. $73-87,2013$ 
Wendt, G. W., Campos, D. M. \& Lisboa, C. S. M. (2010). Agressão entre pares e vitimização no contexto escolar: bullying, cyberbullying e os desafios para a educação contemporânea. Cadernos de Psicopedagogia, 8, 41-52.

Williams, K. \& Guerra, N. (2007). Prevalence and predictors of internet bullying. Journal of Adolescent Health, 41(6), S14-S21.

Recebido em 15 de outubro de 2012

Aceito para publicação em 29 de março de 2013 